\title{
Can cholesterol be used to distinguish pleural exudates from transudates? evidence from a bivariate meta-analysis
}

\author{
Yongchun Shen ${ }^{1+}$, Hong Zhu ${ }^{2+}$, Chun Wan ${ }^{1}$, Lei Chen ${ }^{1}$, Tao Wang ${ }^{1}$, Ting Yang ${ }^{1}$ and Fuqiang Wen ${ }^{1 *}$
}

\begin{abstract}
Background: Many studies have investigated whether pleural cholesterol levels can aid in diagnosis of pleural exudates, and the results have varied considerably. To gain a more reliable answer to this question, we meta-analyzed the literature on using pleural cholesterol or the ratio of cholesterol in pleural fluid to cholesterol in serum (P/S cholesterol ratio) as diagnostic tests to help identify pleural exudates.

Methods: Literature databases were systematically searched for studies examining accuracy of pleural cholesterol or P/S cholesterol ratios for diagnosing pleural exudates. Data on sensitivity, specificity, positive/negative likelihood ratio (PLR/NLR), and diagnostic odds ratio (DOR) were pooled using bivariate-effects models. Summary receiver operating characteristic (SROC) curves and area under the curve (AUC) were used to summarize overall test performance.

Results: Our meta-analysis included up to 20 studies involving 3,496 subjects. Summary estimates for pleural cholesterol in the diagnosis of pleural exudates were as follows: sensitivity, 0.88 (95\%Cl 0.84 to 0.92); specificity, 0.96 ( $95 \%$ Cl 0.92 to 0.98); PLR, 20.31 (95\% Cl 11.21 to 36.78); NLR, 0.12 (95\% Cl 0.09 to 0.17); DOR, 167.06 (95\% Cl 76.79 to 363.95); and AUC 0.97 ( $95 \% \mathrm{Cl} 0.95$ to 0.98 ). The corresponding summary performance estimates for using the P/S cholesterol ratio were as follows: sensitivity, 0.94 ( $95 \%$ Cl 0.92 to 0.96 ); specificity, 0.87 ( $95 \%$ Cl 0.83 to 0.91); PLR 7.46 (95\% Cl, 5.47 to 10.19); NLR, 0.07 ( $95 \%$ Cl 0.05 to 0.10); DOR, 107.74 ( $95 \%$ Cl 60.91 to 190.60); and AUC 0.97 ( $95 \%$ Cl 0.95 to 0.98).

Conclusions: Both pleural cholesterol level and the P/S cholesterol ratio are helpful for the diagnosis of pleural exudates. Nevertheless, the results of pleural cholesterol assays should be interpreted in parallel with the results of traditional tests and clinical information.
\end{abstract}

Keywords: Cholesterol, Exudate, Pleural fluid, Meta-analysis

\section{Background}

Pleural effusion often develops in patients with thoracic or systemic diseases [1]. Such effusion has traditionally been classified as transudate or exudate based on the etiology and underlying pathology, and differentiating the two types of pleural effusion is critical for guiding treatment $[2,3]$. Transudates are usually taken as a sign of underlying congestive heart failure, cirrhosis, or nephrosis, which then becomes the focus of treatment. Because in the case of a transudate, aetiology and therapy are directed

\footnotetext{
* Correspondence: wenfuqiang.scu@gmail.com

${ }^{\dagger}$ Equal contributors

'Division of Pulmonary Diseases, State Key Laboratory of Biotherapy of China and Department of Respiratory and Critical Care Medicine, West China

Hospital of Sichuan University, Chengdu 610041, China

Full list of author information is available at the end of the article
}

to the underlying congestive heart failure, cirrhosis, or nephrosis. Alternatively, exudates are usually taken as a sign of, inflammatory disorders or malignancy, leading to more extensive diagnostic procedures $[4,5]$.

The criteria most widely used to differentiate exudates and transudates in patients with pleural effusions are Light's criteria, established by Light et al. in 1972 [6]. These criteria rely on levels of total protein and of lactate dehydrogenase [6]. The criteria maximize sensitivity at the expense of specificity: they typically identify $98 \%$ of pleural exudates, but they misclassify approximately $25 \%$ of transudates as exudates [7]. As a result, patients misclassified with exudate undergo unnecessary and risky invasive diagnostic procedures, such as thoracoscopic pleural biopsy and image-guided percutaneous pleural 
biopsy [8]. This highlights the need to develop better methods to differentiate exudative and transudative pleural effusions [9].

Since Light's criteria were published in 1972, pleural cholesterol levels have been explored for their usefulness in diagnosing pleural exudates. Cellular degeneration and vascular leakage due to increased permeability are thought to elevate pleural cholesterol levels [10]. Several studies suggest that pleural cholesterol is increased in pleural exudates, making it a potential biomarker for differentiating exudative and transudative pleural effusions [11]. Studies have come to conflicting conclusions about whether pleural cholesterol levels can provide adequate differentiating power, and other studies have explored the diagnostic potential of the ratio of cholesterol in pleural fluid to cholesterol in serum $(\mathrm{P} / \mathrm{S}$ cholesterol ratio) and come to similarly conflicting conclusions [11]. To help gain more reliable insights, we meta-analyzed the literature on using pleural cholesterol level or the $\mathrm{P} / \mathrm{S}$ cholesterol ratio to distinguish pleural exudates from transudates.

\section{Methods}

The present meta-analysis was performed using the guidelines of the Preferred Reporting Items for Systematic Reviews, as well as the Meta-analysis Statement and methods recommended by the Cochrane Diagnostic Test Accuracy Working Group [12]. Institutional review board approval was not required for this retrospective meta-analysis.

Two investigators (YCS and HZ) searched in PUBMED and EMBASE for relevant articles published up to October 2013 in which the following search terms were used as Medical Headings and/or text words: "cholesterol", "pleural effusion", and "pleural fluid". The syntax for the PUBMED searches was as follows: "pleural effusion" OR "pleural fluid" AND "cholesterol". Reference lists of the included studies and review articles were also checked to identify additional studies.

Studies were included if they fulfilled the following criteria: (1) they were original research articles published in English; (2) they examined the ability of pleural cholesterol level or $\mathrm{P} / \mathrm{S}$ cholesterol ratio for differentiating pleural transudates and exudates in humans; and (3) they reported sufficient data to allow calculation of true positive (TP), false positive (FP), false negative (FN), and true negative (TN) rates. Conference proceedings and studies published only as abstracts were excluded. To avoid selection bias, we also excluded studies involving fewer than 20 patients.

Two reviewers (LC and TW) independently identified eligible studies and extracted data, with which they prepared $2 \times 2$ tables of diagnostic performance. In case of disagreement, the two reviewers arrived at a consensus. The quality of the selected studies was assessed using the 14-items Quality Assessment of Diagnostic Accuracy Studies (QUADAS) list [13].

Using bivariate regression, we calculated pooled estimates of sensitivity (SEN) and specificity (SPE) as the main outcome measures, and we constructed summary receiver operating characteristic (SROC) curves [14]. The bivariate approach investigates potential between-study heterogeneity and takes into account possible correlation between SEN and SPE. Based on the pooled estimates of SEN and SPE, we calculated positive likelihood ratios (PLR) and negative likelihood ratios (NLR). SEN and SPE estimates were paired to generate diagnostic odds ratios (DOR), which we used as an overall index of diagnostic accuracy. DOR relates the odds of positive test results in those with the condition with the odds of positive test results in those without the condition.

Heterogeneity was assessed using the $\mathrm{I}^{2}$ inconsistency test. $\mathrm{I}^{2}>50 \%$ indicated substantial heterogeneity, which was then analyzed through subgroup analyses. The following covariates were clearly reported by more than $80 \%$ of included studies and so were analyzed as possible sources of heterogeneity: publication year (before 2000 vs. after 2000), design (cross sectional vs. non-cross sectional), data collection (prospective vs. retrospective), sampling method (consecutive/random vs. nonconsecutive/nonrandom/not reported), blinding (yes vs. no or not reported), detection method (enzymatic colorimetric method vs. other), sample size ( $<100$ subjects vs. $\geq 100$ subjects), QUADAS score $(<9$ vs. $\geq 9)$, and cholesterol cut-off value (60 mg/dl vs. other) [11].

Deeks's funnel plot was used to detect publication bias [15]. Post-test probability (PTP) was calculated using the overall prevalence of $20 \%$ with Fagan nomograms. All analyses were performed using the "Midas" module in STATA 12.0 (Stata Corp., College Station, TX). All statistical tests were two-sided, with $\mathrm{P}$ values less than 0.05 taken as the threshold for statistical significance.

\section{Results}

After systematically searching literature databases and manually searching references lists in relevant reviews and studies, we included 20 studies examining the diagnostic accuracy of cholesterol levels in patients with pleural exudates [16-35]. Studies were excluded mainly because they did not examine diagnostic performance, they did not report sufficient data to construct $2 \times 2$ tables, or they involved fewer than 20 patients. Figure 1 outlines the process of selecting studies.

\section{Quality of reporting and study design}

The final set of 20 studies involved 3,496 subjects, comprising 2,548 patients with pleural exudates and 948 with transudates. Of the included studies, 19 examined the ability of pleural cholesterol concentrations to 


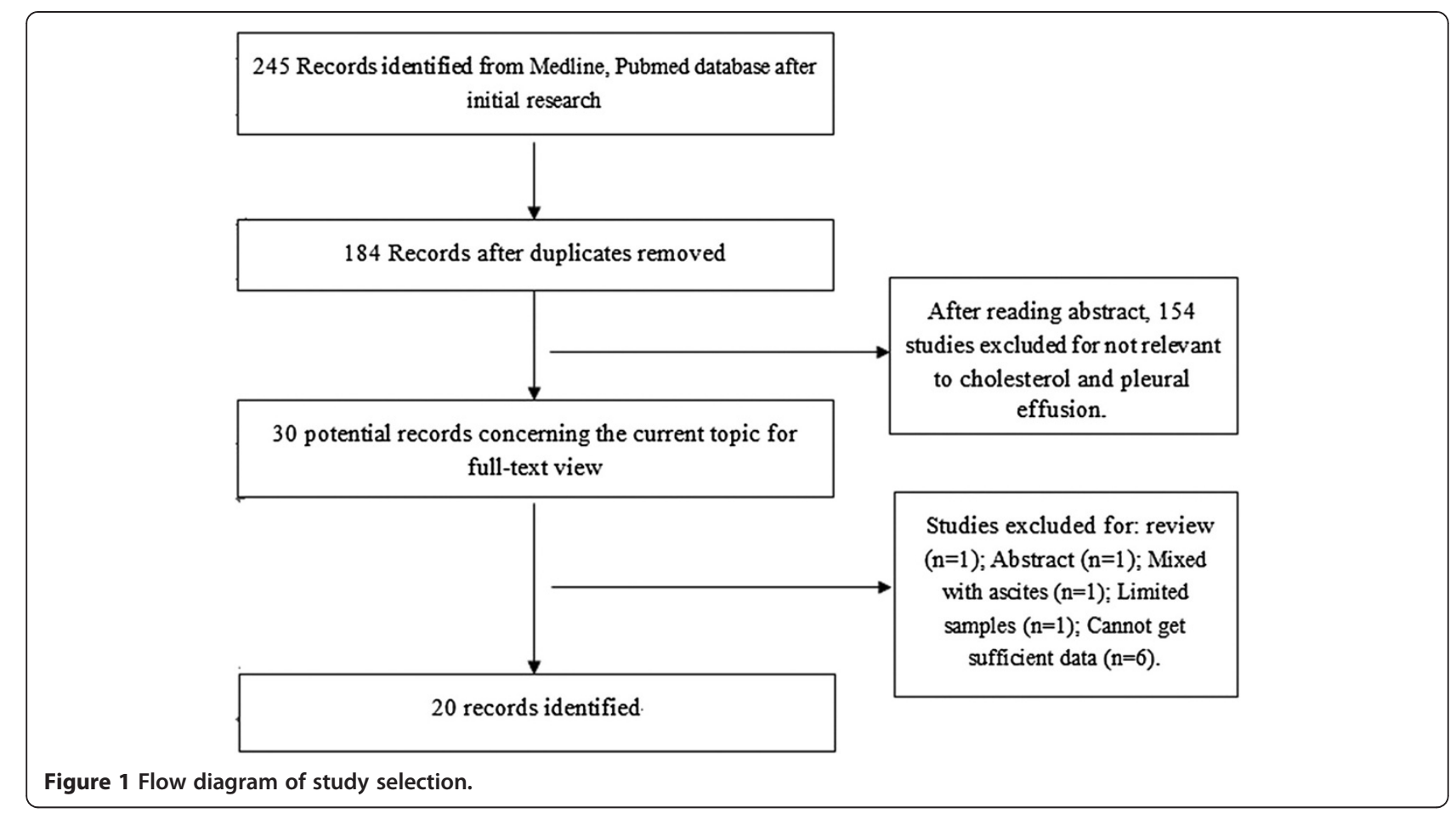

Table 1 Clinical summary of included studies examining the diagnostic accuracy of pleural cholesterol level

\begin{tabular}{|c|c|c|c|c|c|c|c|c|c|c|c|c|c|}
\hline \multirow[t]{2}{*}{ Study (ref no.) } & \multirow[t]{2}{*}{ Year } & \multirow[t]{2}{*}{ Country } & \multicolumn{2}{|c|}{ No. patients } & \multirow[t]{2}{*}{ Standard } & \multirow[t]{2}{*}{ Method } & \multirow{2}{*}{$\begin{array}{l}\text { Cut-off } \\
\text { (mg/l) }\end{array}$} & \multirow[t]{2}{*}{ TP } & \multirow[t]{2}{*}{ FP } & \multirow[t]{2}{*}{ FN } & \multirow[t]{2}{*}{ TN } & \multirow{2}{*}{$\begin{array}{l}\text { QUADAS } \\
\text { score }\end{array}$} & \multirow[t]{2}{*}{ Misclassified } \\
\hline & & & Exu & Trans & & & & & & & & & \\
\hline Hamm H [16] & 1987 & Germany & 31 & 31 & Clinical criteria & ECM & 60 & 28 & 3 & 3 & 28 & 8 & $9 / 30$ \\
\hline Valdés L [17] & 1991 & Spain & 188 & 65 & Clinical criteria & ECM & 55 & 171 & 0 & 17 & 65 & 9 & $14 / 65$ \\
\hline Romero S [18] & 1993 & Spain & 253 & 44 & Clinical criteria & ECM & 60 & 206 & 4 & 47 & 40 & 8 & $10 / 44$ \\
\hline Burgess LJ [19] & 1995 & South Africa & 270 & 123 & Clinical criteria & ESM & 50 & 184 & 11 & 86 & 112 & 7 & $19 / 112$ \\
\hline Costa M [20] & 1995 & Chile & 131 & 49 & Clinical criteria & ECM & 45 & 118 & 0 & 13 & 49 & 9 & $9 / 49$ \\
\hline Gil Suay V [21] & 1995 & Spain & 156 & 48 & Clinical criteria & ECM & 54 & 149 & 4 & 7 & 44 & 8 & $17 / 48$ \\
\hline Garcia-Pachon E [22] & 1996 & Spain & 118 & 35 & Clinical criteria & NA & 50 & 107 & 1 & 11 & 34 & 10 & $9 / 35$ \\
\hline Kalayci AG [23] & 1996 & Turkey & 40 & 20 & Clinical criteria & ECM & 40 & 36 & 1 & 4 & 19 & 9 & NA \\
\hline Metintaş M [24] & 1997 & Turkey & 72 & 21 & Clinical criteria & ECM & 60 & 54 & 3 & 18 & 18 & 10 & $4 / 21$ \\
\hline Gázquez I [25] & 1998 & Spain & 155 & 38 & Clinical criteria & ECM & 50 & 130 & 6 & 25 & 32 & 7 & $11 / 38$ \\
\hline Romero S [26] & 2000 & Spain & 182 & 61 & Clinical criteria & ECM & 60 & 155 & 5 & 27 & 56 & 9 & $9 / 61$ \\
\hline Yilmaz A [27] & 2000 & Turkey & 150 & 105 & Clinical criteria & ECM & 60 & 143 & 4 & 7 & 101 & 8 & $13 / 105$ \\
\hline Porcel JM [28] & 2001 & Spain & 139 & 32 & Clinical criteria & ECM & 60 & 111 & 1 & 28 & 31 & 9 & $8 / 32$ \\
\hline Alexandrakis MG [29] & 2002 & Greece & 82 & 24 & Light's criteria & NA & 65 & 76 & 0 & 6 & 24 & 10 & NA \\
\hline Guleria R [30] & 2003 & India & 50 & 25 & Clinical criteria & NA & 60 & 44 & 0 & 6 & 25 & 9 & $5 / 25$ \\
\hline Leers MP [31] & 2007 & Netherlands & 300 & 108 & Clinical criteria & ECM & 60 & 227 & 2 & 73 & 106 & 11 & 29/108 \\
\hline Razi E [32] & 2008 & Iran & 70 & 49 & Light's criteria & NA & 38 & 61 & 10 & 9 & 39 & 8 & NA \\
\hline Hamal AB [33] & 2013 & Nepal & 43 & 19 & Clinical criteria & ECM & 45 & 42 & 0 & 1 & 19 & 12 & NA \\
\hline Patel AK [34] & 2013 & India & 49 & 11 & Clinical criteria & NA & 60 & 48 & 0 & 1 & 11 & 11 & $0 / 11$ \\
\hline
\end{tabular}

Abbreviations: ECM, enzymatic colorimetric method; ESM, enzymatic spectrophotometric method; Exu, pleural exudate; Trans, transudate; TP, no. of true positives; FP, no. of false positives; FN, no. of false negatives; NA, not applicable; TN, no. of true negatives; QUADAS, quality assessment of diagnostic accuracy studies. *No. of transudates misclassified as exudates based on Light's criteria (misclassifications/total). 
Table 2 Clinical summary of included studies examining the diagnostic accuracy of the ratio of cholesterol in pleural fluid to cholesterol in serum

\begin{tabular}{|c|c|c|c|c|c|c|c|c|}
\hline \multirow[t]{2}{*}{ Study (ref. no.) } & \multirow[t]{2}{*}{ Year } & \multicolumn{2}{|c|}{ Sample size } & \multirow[t]{2}{*}{ Cut-off } & \multirow[t]{2}{*}{ TP } & \multirow[t]{2}{*}{ FP } & \multirow[t]{2}{*}{ FN } & \multirow[t]{2}{*}{ TN } \\
\hline & & Exudates & Transudates & & & & & \\
\hline Hamm H [16] & 1987 & 31 & 31 & 0.3 & 29 & 2 & 2 & 29 \\
\hline Valdés L [17] & 1991 & 188 & 65 & 0.3 & 174 & 8 & 14 & 57 \\
\hline Romero S [18] & 1993 & 249 & 42 & 0.3 & 222 & 12 & 27 & 30 \\
\hline Burgess LJ [19] & 1995 & 270 & 123 & 0.3 & 240 & 23 & 30 & 100 \\
\hline Gil Suay V [21] & 1995 & 156 & 48 & 0.32 & 152 & 4 & 4 & 44 \\
\hline Garcia-Pachon E [22] & 1996 & 118 & 35 & 0.3 & 109 & 1 & 9 & 34 \\
\hline Kalayci AG [23] & 1996 & 40 & 20 & 0.3 & 38 & 2 & 2 & 18 \\
\hline Metintaş M [24] & 1997 & 72 & 21 & 0.3 & 67 & 6 & 5 & 15 \\
\hline Romero S [26] & 2000 & 182 & 61 & 0.3 & 171 & 11 & 11 & 50 \\
\hline Yilmaz A [27] & 2000 & 150 & 105 & 0.3 & 143 & 11 & 7 & 94 \\
\hline Horvath LL [35] & 2001 & 69 & 40 & 0.3 & 68 & 2 & 1 & 38 \\
\hline Alexandrakis MG [29] & 2002 & 82 & 24 & 0.38 & 77 & 3 & 5 & 21 \\
\hline Guleria R [30] & 2003 & 50 & 25 & 0.4 & 49 & 4 & 1 & 21 \\
\hline
\end{tabular}

Abbreviations: TP, no. of true positives; FP, no. of false positives; FN, no. of false negatives; NA, not applicable; TN, no. of true negatives.

distinguish exudates from transudates, while 13 examined the ability of the $\mathrm{P} / \mathrm{S}$ cholesterol ratio to do this. Included studies were published between 1987 and 2013. In all included studies, most studies took into account the limitations of Light's criteria and therefore diagnosed exudative pleural effusion by combining Light's criteria with clinical information, treatment response, and other data, which is widely considered an acceptable basis for exudate diagnosis. Of the 20 studies, 12 had QUADAS scores $\geq 9$. Tables 1 and 2 summarize the clinical characteristics of the patients in each study as well as the QUADAS scores for each publication.

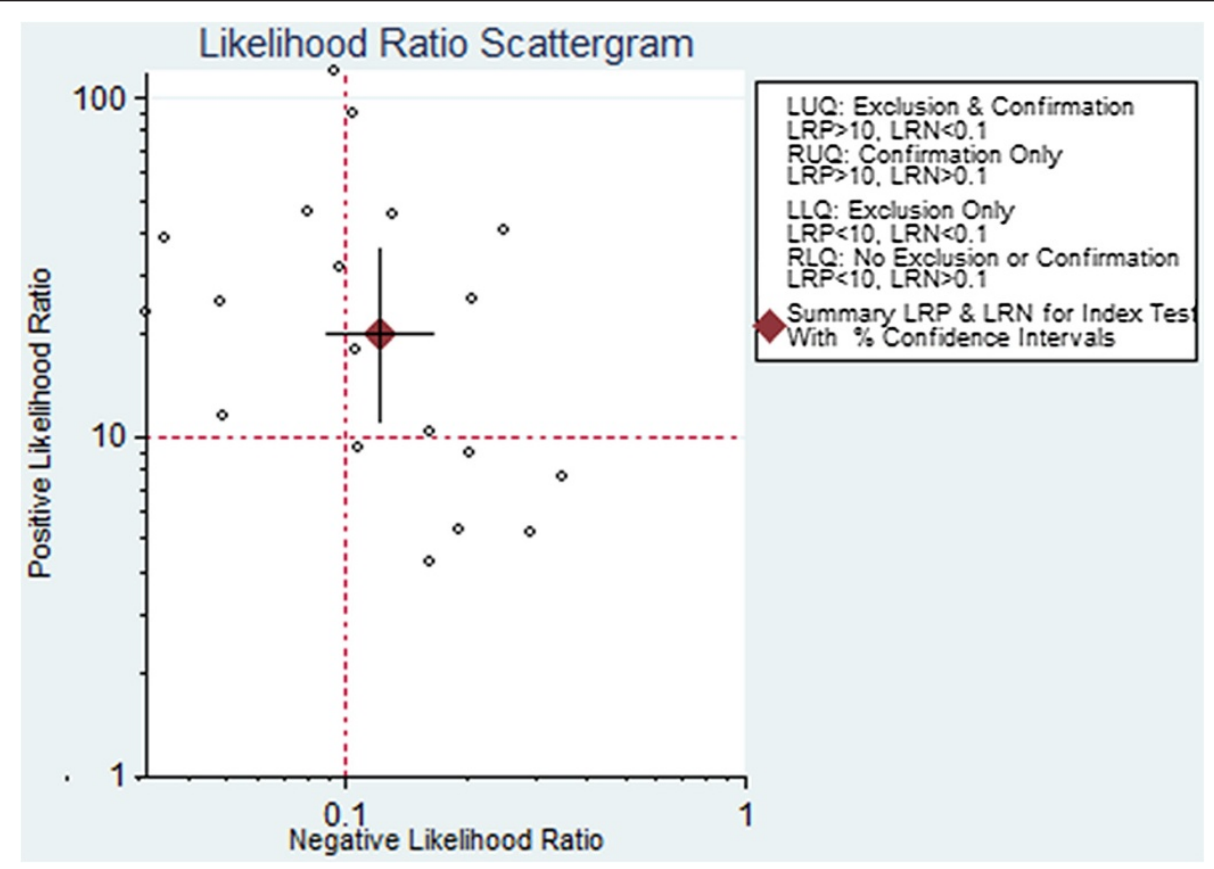

Figure 2 Scatterplot of the positive likelihood ratio (PLR) and negative likelihood ratio (NLR) when using pleural cholesterol concentrations to diagnose exudates. 


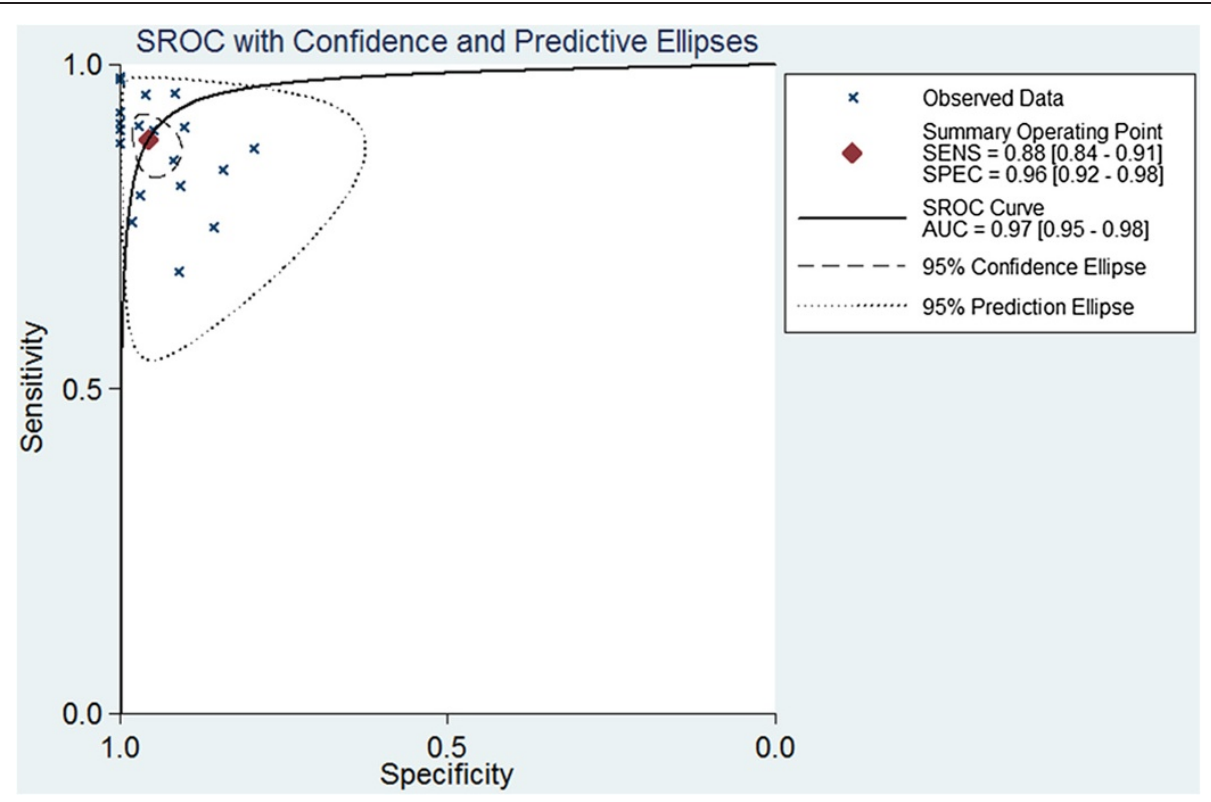

Figure 3 Summary receiver operating characteristic (SROC) curve for pleural cholesterol concentration as a diagnostic test for exudates.

\section{Diagnostic accuracy of pleural cholesterol level}

The following pooled parameters were calculated over all 19 studies examining pleural cholesterol concentrations for diagnosing exudates: SEN, 0.88 (95\% CI: 0.840.91); SPE, 0.96 (95\% CI: 0.92-0.98); PLR, 20.31 (95\% CI: 11.21-36.78) (Figure 2); NLR, 0.12 (95\% CI: 0.09-0.17) (Figure 2); and DOR, 167.06 (95\% CI: 76.69-363.95). All five performance indices showed high $\mathrm{I}^{2}$ values: SEN, 91.13\%; SPE, 74.31\%; PLR, 63.7\%; NLR, 91.96\%; and DOR, $100 \%$ (all $\mathrm{P}<0.05$ ). This suggests substantial heterogeneity among the studies.

Figure 3 shows a plot of the rate of true positives as a function of the rate of false positives for individual studies, as well as the corresponding SROC curve. Using the bivariate approach, which estimates not only the strength but also the shape of the correlation between SEN and SPE, we plotted the observed and predicted ellipses at a 95\% confidence level. The AUC was 0.97 (95\% CI: 0.95-0.98), indicating a high discriminatory ability of pleural cholesterol. Fagan's nomogram for likelihood ratios (Figure 4) indicated that using cholesterol to detect pleural exudates increased the post-probability to $83 \%$ when the results were positive and reduced the post-probability to $4 \%$ when the results were negative.

\section{Diagnostic accuracy of the P/S cholesterol ratio}

A total of 13 studies with 2,297 subjects examined the ability of the $\mathrm{P} / \mathrm{S}$ cholesterol ratio to distinguish exudates from transudates. Table 3 summarizes the SEN, SPE, PLR, NLR, and DOR, while Figure 5 shows the SROC curve. The AUC was 0.97 (95\% CI: 0.95-0.98), suggesting high overall accuracy.

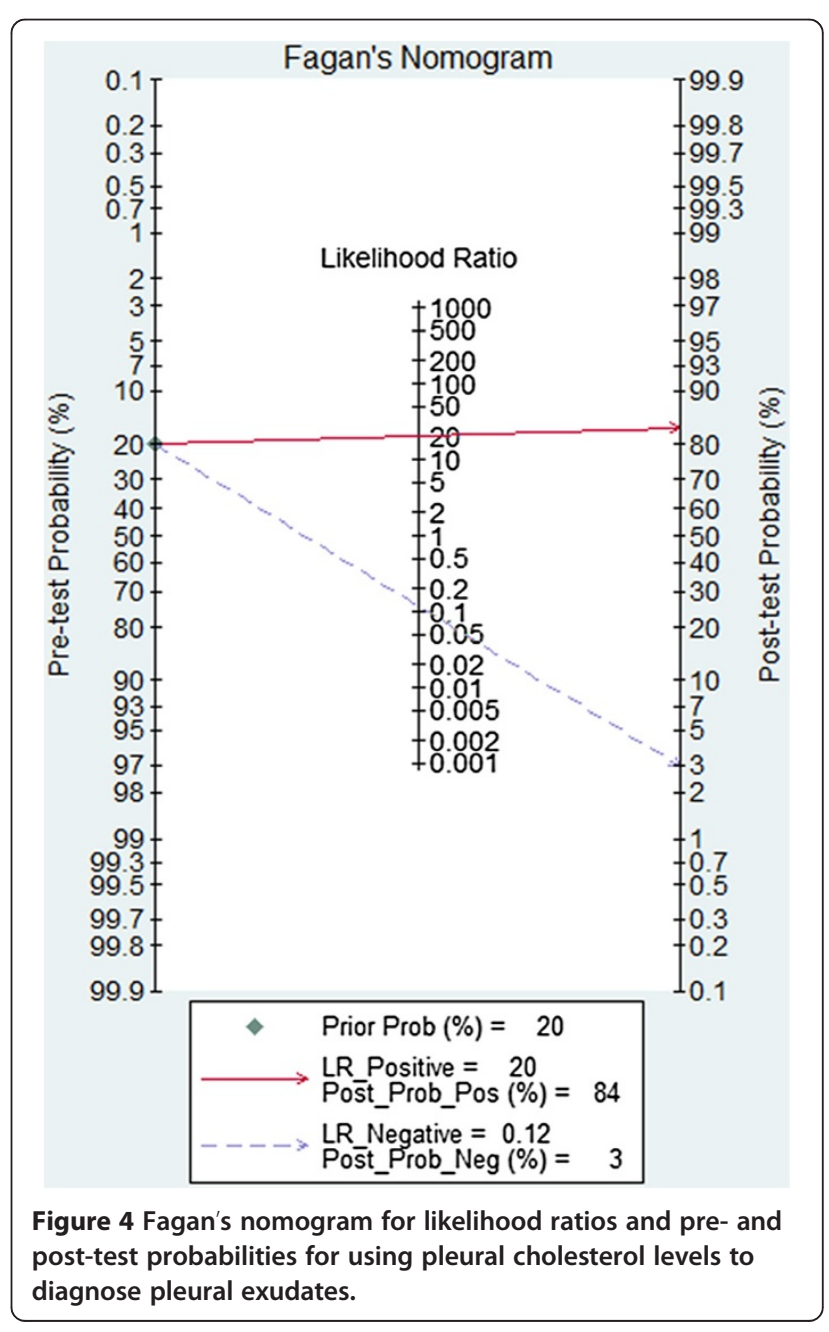


Table 3 Summary characteristics of diagnostic performance of pleural cholesterol levels and ratio of cholesterol in pleural fluid to cholesterol in serum

\begin{tabular}{lll}
\hline Parameter & $\begin{array}{l}\text { Pleural cholesterol } \\
\text { concentrations }\end{array}$ & $\begin{array}{l}\text { Pleural fluid to serum } \\
\text { cholesterol ratio }\end{array}$ \\
\hline Sensitivity & 0.88 & 0.94 \\
Specificity & $(95 \mathrm{Cl}: 0.84-0.92)$ & $(95 \mathrm{Cl}: 0.92-0.96)$ \\
& 0.96 & 0.87 \\
PLR & $(95 \mathrm{Cl}: 0.92-0.98)$ & $(95 \mathrm{Cl}: 0.83-0.91)$ \\
& 20.31 & 7.46 \\
NLR & $(95 \mathrm{Cl}: 11.21-36.78)$ & $(95 \mathrm{Cl}: 5.47-10.19)$ \\
& 0.12 & 0.07 \\
DOR & $(95 \mathrm{Cl}: 0.09-0.17)$ & $(95 \mathrm{Cl}: 0.05-0.10)$ \\
& 167.06 & 107.74 \\
PPP & $(95 \mathrm{Cl}: 76.69-363.95)$ & $(95 \mathrm{Cl}: 60.91-190.60)$ \\
PPN & $84 \%$ & $65 \%$ \\
AUC & $3 \%$ & $2 \%$ \\
& 0.97 & 0.97 \\
\hline
\end{tabular}

\section{Investigations of heterogeneity and publication bias}

Significant heterogeneity was identified among included studies, so we performed subgroup analysis to investigate the possible sources of this heterogeneity. Table 4 summarizes the influence of certain covariates on the SEN and SPE. SPE was significantly higher with some covariates, such as QUADAS score or blinding. However, these covariates did not significantly affect SEN.
Deeks' funnel plot asymmetry test was used to assess likelihood of publication bias in the final set of studies. The slope coefficient was associated with a $\mathrm{P}$ value of 0.50 , suggesting symmetry in the data and low likelihood of such bias (Figure 6).

\section{Discussion}

Despite the importance of defining the type of pleural effusion accurately, current methods lack the power to reliably differentiate pleural exudates from transudates [7]. Several studies suggest that pleural cholesterol levels and the $\mathrm{P} / \mathrm{S}$ cholesterol ratio may be able to accomplish this, but the studies have given conflicting results. Our metaanalysis suggests that both pleural cholesterol level and $\mathrm{P} / \mathrm{S}$ cholesterol ratio are useful as tools in the diagnosis of pleural exudates, though they probably cannot stand on their own and so should be used in conjunction with more traditional tests.

Our meta-analysis showed that pleural cholesterol level was associated with high SEN (0.88, 95\% CI: 0.84$0.91)$ and SPE (0.96, 95\% CI: 0.92-0.98). These findings suggest that pleural cholesterol may represent a new milestone in pleural exudate diagnosis. The SROC curve illustrates overall test performance, and shows the tradeoff between SEN and SPE. Our SROC analysis showed an AUC of 0.97, suggesting high overall accuracy. Another indicator of diagnostic accuracy is DOR, which is the ratio of the odds of a true positive to the odds of a false positive; DOR combines SEN and SPE data into a single number ranging from 0 to infinity, with higher values indicating better discriminatory test performance.

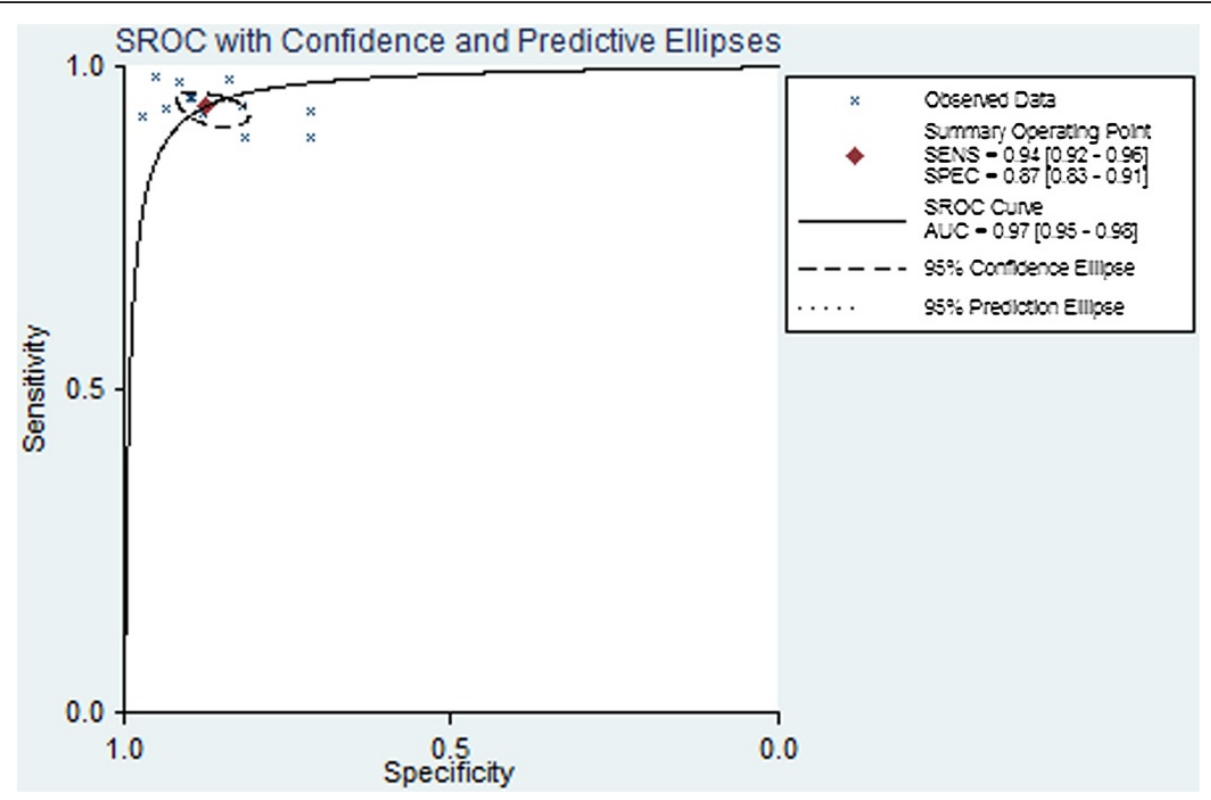

Figure 5 Summary receiver operating characteristic (SROC) curve for using the ratio of cholesterol in pleural fluid to cholesterol in serum to diagnose pleural exudates. 
Table 4 Subgroup analysis for sensitivity and specificity

\begin{tabular}{|c|c|c|c|c|c|c|c|c|}
\hline Covariate & Sensitivity & Coefficient & $z$ & $\mathbf{P}$ & Specificity & Coefficient & $Z$ & $\mathbf{P}$ \\
\hline Publication year & $0.90(95 \%$ Cl 0.84-0.94) & 2.19 & 0.84 & 0.40 & $0.97(95 \%$ Cl 0.92-0.99) & 3.39 & 0.93 & 0.35 \\
\hline Method & $0.88(95 \%$ Cl 0.83-0.92) & 2.01 & -0.20 & 0.84 & $0.96(95 \%$ Cl 0.92-0.98) & 3.07 & -0.10 & 0.92 \\
\hline Cut-off value & $0.86(95 \%$ Cl 0.80-0.91) & 1.85 & -0.96 & 0.34 & $0.96(95 \%$ Cl 0.91-0.98) & 3.08 & -0.02 & 0.99 \\
\hline QUADAS score & $0.89(95 \%$ Cl 0.84-0.93) & 2.07 & 0.34 & 0.74 & $0.98(95 \%$ Cl 0.95-0.99) & 3.77 & 3.15 & 0.00 \\
\hline Sample size & $0.87(95 \%$ Cl 0.82-0.91) & 1.92 & -1.03 & 0.30 & 0.95 (95\% Cl 0.91-0.98) & 3.02 & -0.36 & 0.72 \\
\hline Prospective & $0.90(95 \%$ Cl 0.85-0.93) & 2.18 & 1.08 & 0.28 & 0.95 (95\% Cl 0.90-0.98) & 2.95 & -0.64 & 0.52 \\
\hline Design & $0.90(95 \%$ Cl 0.86-0.93) & 2.22 & 1.70 & 0.09 & $0.94(95 \%$ Cl 0.90-0.97) & 2.81 & -1.42 & 0.16 \\
\hline Consecutive & $0.87(95 \%$ Cl 0.82-0.91) & 1.90 & -1.50 & 0.13 & $0.95(95 \%$ Cl 0.91-0.97) & 2.87 & -1.56 & 0.12 \\
\hline Blinding & 0.88 (95\% Cl 0.80-0.93) & 1.99 & -0.16 & 0.88 & $0.98(95 \%$ Cl 0.95-0.99) & 3.95 & 2.00 & 0.05 \\
\hline
\end{tabular}

Mean DOR in our meta-analysis was 167.06, suggesting that assaying pleural cholesterol levels should be helpful in the diagnosis of pleural exudates.

We further examined the diagnostic accuracy of pleural cholesterol levels by calculating PLR and NLR, which can be easier to relate to clinical practice than SROC and DOR [36]. The pooled PLR value of 20.31 suggests that patients with pleural exudates have an approximately 20fold higher chance of giving a positive pleural cholesterol test result than do patients without exudates. At the same time, the pooled NLR was 0.12 , indicating that a negative pleural cholesterol test result is $12 \%$ likely to be a false negative, which is not low enough to rule out pleural exudates. This comprehensive analysis of the diagnostic accuracy of pleural cholesterol levels suggests that this indicator may not be reliable enough on its own but should instead be used in conjunction with other conventional tests. This unreliability may in part reflect the sensitivity of pleural cholesterol levels to differences in disease conditions, patient population, and environment.

Using the $\mathrm{P} / \mathrm{S}$ cholesterol ratio instead of absolute cholesterol levels may help eliminate the effects of such factors to aid the diagnosis of pleural exudates. Our results revealed that $\mathrm{P} / \mathrm{S}$ cholesterol ratio showed even higher SEN than pleural cholesterol levels (0.94, 95\% CI: 0.92-0.96); in addition, the AUC was 0.97, suggesting that the $\mathrm{P} / \mathrm{S}$ cholesterol ratio also shows high discriminatory ability.

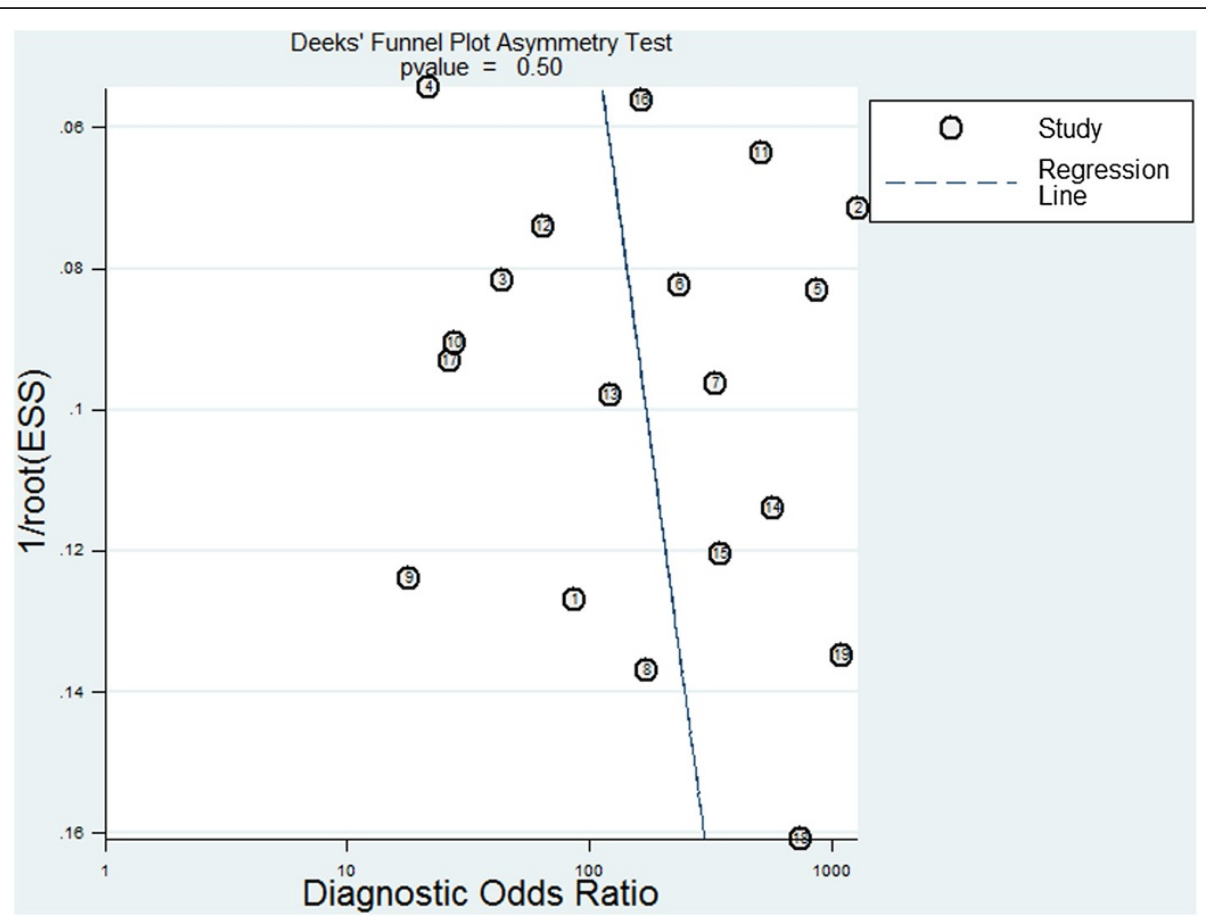

Figure 6 Deek's funnel plot to assess the likelihood of publication bias. 
Misclassification of transudates as exudates can lead to inappropriate patient management or potentially unnecessary and invasive diagnostic investigations that increase morbidity and health care costs [37]. Therefore we compared how often clinicians misclassified pleural transudates as exudates depending on whether they relied on pleural cholesterol levels or Light's criteria (Table 1). Pleural cholesterol levels were associated with a significantly lower misclassification rate. We conclude that pleural cholesterol level shows substantial promise as a supplementary test for distinguishing pleural exudates from transudates.

While meta-analysis is well-suited for generating summary outcomes, the pooled results can mask heterogeneity that should be understood in detail [38]. Indeed we detected substantial heterogeneity across the included studies, and subgroup analyses suggest that differences in QUADAS score accounted for most of the observed SPE heterogeneity. Such differences did not, however, significantly affect SEN, suggesting that study quality had little influence on the TP rate of pleural cholesterol tests. Given the effect of study quality on SPE and the low QUADAS scores of several included studies, future studies should aim for greater rigor in order to decrease the risk of bias.

Our meta-analysis suggests an association between elevated pleural cholesterol and the presence of pleural exudates, which implies that cholesterol contributes to exudate pathogenesis. It is not immediately clear how this happens, so future research should examine this question in order to provide a biological basis for the observed association. Our meta-analysis also points out the need for investigating the effect of cut-off value on the diagnostic accuracy of pleural cholesterol levels. The values in our meta-analysis ranged from 38 to $65 \mathrm{mg} / \mathrm{dl}$. This variation in cut-off value partly reflects differences in clinical context: a lower cut-off value is typically used for cardiac patients with pleural effusion than for patients in cancer institutes. Further work should aim to identify the cut-off value that provides optimal diagnostic accuracy [11].

The findings of this meta-analysis should be interpreted with caution because of several limitations. While our strict inclusion and exclusion criteria may have helped reduce selection bias, they led to a relatively small final set of studies for which statistical power may be inadequate for drawing definitive conclusions about the ability of pleural cholesterol levels to discriminate between exudates and transudates. For example, we included only studies published in English in a relatively small number of databases. Our results may be biased by our omission of unpublished studies, studies published in other languages and studies published in journals not indexed in the databases we searched.

\section{Conclusions}

Our meta-analysis suggests that assaying pleural cholesterol levels and the $\mathrm{P} / \mathrm{S}$ cholesterol ratio may significantly aid the diagnosis of pleural exudates. In the near future, cholesterol-based assays may prove useful as a non-invasive confirmatory test to complement current screening procedures and as a rapid clinical test to guide the comprehensive management of patients with pleural effusion.

\begin{abstract}
Abbreviations
$\mathrm{P} / \mathrm{S}$ cholesterol ratio: Ratio of cholesterol level in pleural fluid to cholesterol level in serum; TP: True positive; FP: False positive; FN: False negative; TN: True negative; QUADAS: Quality assessment of diagnostic accuracy studies; SEN: Sensitivity; SPE: Specificity; SROC: Summary receiver operating characteristic; PLR: Positive likelihood ratio; NLR: Negative likelihood ratio; DOR: Diagnostic odds ratio; AUC: Area under the curve.
\end{abstract}

\section{Competing interests}

All authors declare that they have no conflicts of interest or financial disclosures.

\section{Authors' contributions}

YCS and HZ: conceived the article and contributed to the systematic review, meta-analysis, and manuscript writing. LC and Tao: contributed to the systematic review and manuscript writing. CW and TY: contributed to the systematic review and manuscript writing. FQW: guarantor of the manuscript who takes responsibility for the integrity of the work as a whole, from inception to published article. All authors read and approved the final manuscript.

\section{Acknowledgments}

This work was supported by grants 81230001,81301962 and 8130032 from the National Natural Science Foundation of China. We are indebted to the authors of the primary studies included in this meta-analysis; without their contributions, this work would not have been possible.

\section{Author details}

${ }^{1}$ Division of Pulmonary Diseases, State Key Laboratory of Biotherapy of China and Department of Respiratory and Critical Care Medicine, West China Hospital of Sichuan University, Chengdu 610041, China. ${ }^{2}$ Department of Abdominal Cancer, West China Hospital of Sichuan University, Chengdu 610041, China.

Received: 16 January 2014 Accepted: 8 April 2014

Published: 15 April 2014

\section{References}

1. Fisk M, Branley H: Pleural effusion. Br J Hosp Med 2013, 74:C50-C54.

2. Light RW: Pleural effusions. Med Clin North Am 2011, 95:1055-1070.

3. Sahn SA: Getting the most from pleural fluid analysis. Respirology 2012 , 17:270-277.

4. Kruger D: Evaluating the adult with new-onset pleural effusion. JAAPA 2013, 26:20-27.

5. McGrath EE, Anderson PB: Diagnosis of pleural effusion: a systematic approach. Am J Crit Care 2011, 20:119-127.

6. Light RW, Macgregor MI, Luchsinger PC, Ball WC Jr: Pleural effusions: the diagnostic separation of transudates and exudates. Ann Intern Med 1972, 77:507-513.

7. Porcel JM: Identifying transudates misclassified by Light's criteria. Curr Opin Pulm Med 2013, 19:362-367.

8. Shen YC, Liu MQ, Wan C, Chen L, Wang T, Wen FQ: Diagnostic accuracy of vascular endothelial growth factor for malignant pleural effusion: $A$ meta-analysis. Exp Ther Med 2012, 3:1072-1076.

9. Kummerfeldt CE, Chiuzan CC, Huggins JT, Divietro ML, Nestor JE, Sahn SA, Doelken P: Improving the predictive accuracy of identifying exudative effusions. Chest 2013, 145:586-592.

10. Hillerdal G: Chyliform (cholesterol) pleural effusion. Chest 1985, $88: 426-428$. 
11. Heffner JE, Brown $L K$, Barbieri CA: Diagnostic value of tests that discriminate between exudative and transudative pleural effusions. Chest 1997, 111:970-980.

12. Leeflang MM, Deeks JJ, Gatsonis C, Bossuyt PM, Cochrane Diagnostic Test Accuracy Working Group: Systematic reviews of diagnostic test accuracy. Ann Intern Med 2008, 149:889-897.

13. Whiting PF, Weswood ME, Rutjes AW, Reitsma JB, Bossuyt PN, Kleijnen J: Evaluation of QUADAS, a tool for the quality assessment of diagnostic accuracy studies. BMC Med Res Methodol 2006, 6:9.

14. Reitsma JB, Glas AS, Rutjes AW, Scholten RJ, Bossuyt PM, Zwinderman AH: Bivariate analysis of sensitivity and specificity produces informative summary measures in diagnostic reviews. J Clin Epidemiol 2005, 58:982-990.

15. Deeks JJ, Macaskill P, Irwig L: The performance of tests of publication bias and other sample size effects in systematic reviews of diagnostic test accuracy was assessed. J Clin Epidemiol 2005, 58:882-893.

16. Hamm H, Brohan U, Bohmer R, Missmahl HP: Cholesterol in pleural effusions. A diagnostic aid. Chest 1987, 92:296-302.

17. Valdés L, Pose A, Suàrez J, Gonzalez-Juanatey JR, Sarandeses A, San José E, Alvarez Dobaña JM, Salgueiro M, Rodríguez Suárez JR: Cholesterol: a useful parameter for distinguishing between pleural exudates and transudates. Chest 1991, 99:1097-1102.

18. Romero S, Candela A, Martín C, Hernández L, Trigo C, Gil J: Evaluation of different criteria for the separation of pleural transudates from exudates. Chest 1993, 104:399-404

19. Burgess $\sqcup$, Maritz FJ, Taljaard JJ: Comparative analysis of the biochemical parameters used to distinguish between pleural transudates and exudates. Chest 1995, 107:1604-1609.

20. Costa M, Quiroga T, Cruz E: Measurement of pleural fluid cholesterol and lactate dehydrogenase. A simple and accurate set of indicators for separating exudates from transudates. Chest 1995, 108:1260-1263.

21. Gil Suay V, Martínez Moragón E, Cases Viedma E, Perpiñá Tordera M, León Fábregas M, Sanchis Aldás J: Pleural cholesterol in differentiating transudates and exudates. A prospective study of 232 cases. Respiration 1995, 62:57-63.

22. Garcia-Pachon E, Padilla-Navas I, Sanchez JF, Jimenez B, Custardoy J: Pleural fluid to serum cholinesterase ratio for the separation of transudates and exudates. Chest 1996, 110:97-101.

23. Kalayci AG, Gürses N, Adam B, Albayrak D: Significance of pleural fluid cholesterol and beta-2 microglobulin levels for the differentiation of pleural effusions in childhood. Clin Pediatr (Phila) 1996, 35:353-358.

24. Metintaş M, Alataş O, Alataş F, Colak O, Ozdemir N, Erginel S: Comparative analysis of biochemical parameters for differentiation of pleural exudates from transudates Light's criteria, cholesterol, bilirubin, albumin gradient, alkaline phosphatase, creatine kinase, and uric acid. Clin Chim Acta 1997, 264:149-162.

25. Gázquez I, Porcel JM, Vives M, Vicente De Vera MC, Rubio M, Rivas MC: Comparative analysis of Light's criteria and other biochemical parameters for distinguishing transudates from exudates. Respir Med 1998, 92:762-765.

26. Romero S, Martinez A, Hernandez L, Fernandez C, Espasa A, Candela A, Martin C: Light's criteria revisited: consistency and comparison with new proposed alternative criteria for separating pleural transudates from exudates. Respiration 2000, 67:18-23.

27. Yilmaz A, Tunaboyu IK, Akkaya E, Bayramgürler B: A comparative analysis of the biochemical parameters used to distinguish between pleural exudates and transudates. Respirology 2000, 5:363-367.

28. Porcel JM, Vives M, Vicente De Vera MC, Cao G, Rubio M, Rivas MC: Useful tests on pleural fluid that distinguish transudates from exudates. Ann Clin Biochem 2001, 38:671-675.

29. Alexandrakis MG, Kyriakou D, Alexandraki R, Pappa KA, Antonakis N, Bouros $D$ : Pleural interleukin-1 beta in differentiating transudates and exudates: comparative analysis with other biochemical parameters. Respiration 2002, 69:201-206.

30. Guleria R, Agarwal SR, Sinha S, Pande JN, Misra A: Role of pleural fluid cholesterol in differentiating transudative from exudative pleural effusion. Natl Med J India 2003, 16:64-69.

31. Leers MP, Kleinveld HA, Scharnhorst V: Differentiating transudative from exudative pleural effusion: should we measure effusion cholesterol dehydrogenase? Clin Chem Lab Med 2007, 45:1332-1338.
32. Razi E, Moosavi GA, Fakharian E, Abedi M: Relationship of Pleural and Serum Cholesterol and Lipoprotein Levels in Exudative and Transudative Effusions. Tanaffos 2008, 7:37-43.

33. Hamal AB, Yogi KN, Bam N, Das SK, Karn R: Pleural fluid cholesterol in differentiating exudative and transudative pleural effusion. Pulm Med 2013, 2013:135036.

34. Patel AK, Choudhury S: Combined pleural fluid cholesterol and total protein in differentiation of exudates and transudates. Indian J Chest Dis Allied Sci 2013, 55:21-23.

35. Horvath LL, Gallup RA, Worley BD, Merrill GA, Morris MJ: Soluble leukocyte selectin in the analysis of pleural effusions. Chest 2001, 120:362-368

36. Akobeng AK: Understanding diagnostic tests 2: likelihood ratios, pre- and post-test probabilities and their use in clinical practice. Acta Paediatr 2007, 96:487-491.

37. Bielsa S, Porcel JM, Castellote J, Mas E, Esquerda A, Light RW: Solving the Light's criteria misclassification rate of cardiac and hepatic transudates. Respirology 2012, 17:721-726.

38. Song F, Sheldon TA, Sutton AJ, Abrams KR, Jones DR: Methods for exploring heterogeneity in meta-analysis. Eval Health Prof 2001, 24:126-151.

doi:10.1186/1471-2466-14-61

Cite this article as: Shen et al:: Can cholesterol be used to distinguish pleural exudates from transudates? evidence from a bivariate metaanalysis. BMC Pulmonary Medicine 2014 14:61.

\section{Submit your next manuscript to BioMed Central and take full advantage of:}

- Convenient online submission

- Thorough peer review

- No space constraints or color figure charges

- Immediate publication on acceptance

- Inclusion in PubMed, CAS, Scopus and Google Scholar

- Research which is freely available for redistribution 